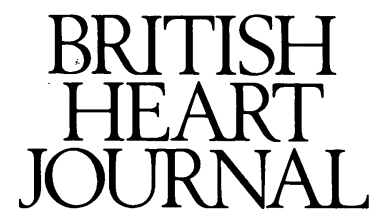

Editorial

\title{
Management of patients with Björk-Shiley prosthetic valves
}

There are three basic types of Björk-Shiley valve (figure) that may be encountered in British patients. The much publicised hazard of strut fracture is effectively confined to the convexo-concave valve so in advising and managing patients electively it is helpful to be able to identify these valves. To some extent the identity of the valve can be determined from its date of implantation, but valves can be distinguished with certainty by their serial numbers when these are available. Otherwise it should be possible to identify them by radiological appearances.

\section{Earlier valves}

The original valve with a Delrin disc was implanted in about 85000 patients between 1969 and $1979.1^{12}$ This was replaced with a pyrolitic carbon disc, known as a "spherical disc", that had a $60^{\circ}$ opening angle. About 180000 of these valves were implanted up to 1987 . The serial numbers carry the prefix ABP for aortic valves and MBRP, MBUP, and MBP for mitral valves, where the first two initials (AB or MB) denote site and Björk design and the last initial (P) identifies the spherical disc pattern. (The $U$ and $R$ refer to details of the relation of the sewing ring to the annulus.) The two struts, which are visible radiologically, can be seen to overlap when the valve is imaged en face (Fig 1A). By 1979 there were only three reports of the disc escaping, out of a total of 90000 implants. These were probably related to damage to the struts at the time of implantation. ${ }^{1}$ Long term follow up in a large series was free of mechanical failure. $^{3}$

The convexo-concave valve

Between 1976 and 1986 the convexo-concave (CC) valve was implanted. ${ }^{24}$ In 1979 the new design was approved by the USA Food and Drugs Administration (FDA)..$^{5}$ It was introduced in an attempt to improve the flow characteristics

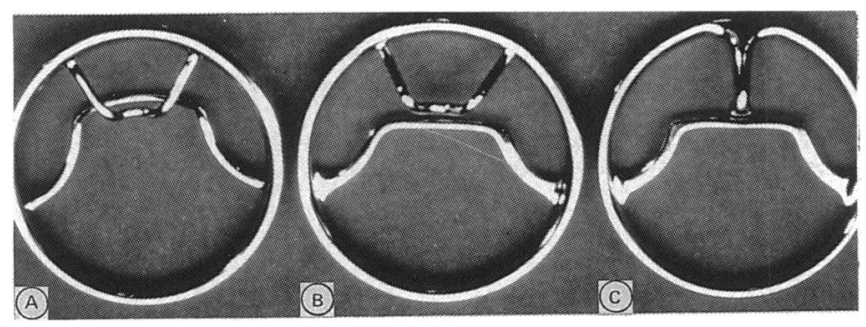

Metallic component of the Björk-Shiley tilting disc valves. (A) The spherical model with "overlapping" struts when seen en face. Strut fracture extremely rare. (B) The convexo-concave valve. These are the valves at risk, particularly in the mitral site. (C) The monostrut Björk-Shiley tilting disc valve. No mechanical failures reported. across the valve and reduce the risk of thrombus formation. The new $60^{\circ}$ convexo-concave valve was implanted in about 82000 patients before being withdrawn in April 1985 by Shiley. Case reports of strut fractures ${ }^{6-8}$ include photographs of a consistent problem. The smaller strut had become detached from the annulus at the site of a weld and the disc escaped. In the meantime Shiley experimented with a $70^{\circ}$ version of the same valve which unfortunately was even more prone to this problem. About one in eight of these valves was likely to fracture within seven years of implantation. ${ }^{5}$ It was never released in the United States but about 4000 were implanted elsewhere between 1980 and $1983 .{ }^{2}$ The codes for the CC valves are ABC, MBRC, $M B U C$, and $M B C$; again the final letter indicates the disc pattern and the numbers 60 or 70 denote the opening angle. The struts do not overlap when the valve is seen en face (Fig 1B). The valves particularly at risk are the $29 \mathrm{~mm}$, $31 \mathrm{~mm}$, and $33 \mathrm{~mm}$ mitral valves manufactured between February 1981 and June 1982. The overall risk of strut fracture is said to be $0 \cdot 295 \%$ per annum. ${ }^{9}$ More detailed estimates have been made by size and postoperative year ${ }^{2}$ and are under $0.1 \%$ per annum in the low risk categories. These are likely to be underestimates because rapid deterioration or sudden death may well be attributed to other cardiac or non-cardiac causes and strut fracture will go undiagnosed.

\section{The monostrut valve}

The monostrut valve was designed to overcome the strut fracture problem and seems to have done so successfully. It was introduced on to the market in 1983 but has never had FDA approval and is not available in the United States. The metal ring with its retaining struts is milled from a single piece. It can readily be identified on a penetrated $x$ ray. The serial numbers begin with the letters ABMS, MBMS, and MBRMS; MS denotes monostrut.

\section{The future}

While it is easy to be wise after the event, it is hard to draw any firm conclusions for the future from this experience. Cardiac surgery is now routine and commonplace and patients and their physicians have come to expect perfect results. But there are many things that can go wrong and with long life expectancy after valve replacement many years in which they can go wrong. The United Kingdom Heart Valve Registry provides a mechanism by which a pattern of sudden death in valve recipients can be detected early. ${ }^{10}$ Of course bench testing should be thorough before clinical use of a new device is contemplated and every care should be taken to do meticulous clinical follow up but because most patients who have heart valve replacements 
are expected to survive for more than 15 years $^{311}$ sooner or later fair wear and tear will take its toll of mechanical devices performing 42 million cycles per year. The annual incidence of problems related to the mechanical valve itself is minute in comparison with the number of patients who die each year of endocarditis, valve thrombosis, embolism, anticoagulant related haemorrhage, and natural progression of disease in the heart and elsewhere. ${ }^{12}$ Virtually all mechanical valve recipients will die with an intact prosthesis. Without innovation we cannot make further progress and yet we know that it has now become extremely difficult for anyone to pioneer a new valve or even a small modification of an old one. If "the industry is so punished by the press and professional critics that innovation is stifled and the risk for failure becomes too great to continue" then we will see no further progress in valve design. This situation seems to be upon us in the USA. ${ }^{13}$

The more immediate problem is how should a clinician deal with patients who have these valves in place. The first obvious piece of advice is that in any patient with an artificial valve who deteriorates, first suspect the valve. If strut fracture has occurred the only hope is emergency reoperation so no time should be lost in referring the patient to the nearest cardiac surgical unit. Strut fracture can be diagnosed by screening alone.

The commoner problem is the large number of patients who remain well but are afraid. First of all the valve should be identified by serial number and if this is not available screening may be justified to identify spherical and monostrut valves which are not prone to this problem, so that the patient can be given this reassuring news without delay. Symptoms and clinical change must be dealt with on their merits but the individual can be strongly reassured about the theoretical risk of strut fracture in the future. ${ }^{9}$ For patients with a convexo-concave valve, while action may not be justified, reassurance must be a little more guarded because one in 30 of those with a mitral valve that is $29 \mathrm{~mm}$ or larger is at risk of death due to strut fracture. The alternative of electively replacing the valve carries a far worse risk. The risk of death with reoperation on the mitral valve was nearly $16 \%$ in 206 cases operated on in United Kingdom units in 1989 (70\% confidence interval 13\% to $19 \%$ ), that is more than three times that of a first operation (J Cleland. United Kingdom Cardiac Surgical Register, personal communication). Reoperation doubles the risk in aortic valve replacement from just over $4 \%$ to nearly $9 \%$ ( $70 \%$ CI $6 \%$ to $12 \%$ ). Of course it could be argued that these figures are those for clinically indicated reoperation including patients with catastrophic states such as endocarditis or sudden valve failure. Nevertheless, the difference between the risk of strut fracture and that of repeat valve surgery is sufficiently great that elective replacement of these valves for fear alone is unjustifiable.

And what is the legal position? Shiley have publicly indicated their willingness to meet claims from patients who have experienced strut fractures and they have settled directly to avoid protracted legal cases. Whether this is a generous and humane gesture or an economically sound strategy is open to interpretation but we all know that the law is a slow and bitter way of gaining compensation for medical injury. On the other hand Shiley will "vigorously defend" anxiety claims and believe that a "functioning valve is no basis for a claim".

I thank Mr J Cleland for supplying figures from the United Kingdom Cardiac Surgical Register and Mr Philip Ayers of Shiley for providing illustrations and technical data.

St George's Hospital,

TOM TREASURE

Blackshaw Road

London SW17 OQT

1 Björk VO, Henze A. Ten years' experience with the Björk-Shiley tilting disc valve. $J$ Thorac Cardiovasc Surg 1979;78:331-42.

Hiratzka LF, Kouchoukos NT, Grunkemeier GL, Miller DC, Scully HE, Wechsler AS. Outlet strut fracture of the Björk-Shiley $60^{\circ}$ convexoconcave valve: current information and recommendations for patient care. J Am Coll Cardiol 1988;11:1130-7.

3 Bloomfield P, Wheatley DJ, Prescott RJ, Miller HC. Twelve-year comparison of a Björk-Shiley mechanical heart valve with porcine bioprostheses. N Engl J Med 1991;324:573-9.

4 Nightingale SL. Shiley heart valve physician and patient notification program. JAMA 1991;265:847.

5 Brahams D. Björk-Shiley heart-valve failures. Lancet 1990;336:619-20.

6 Sethia B, Quin RO, Bain WH. Disc embolisation after minor strut fracture in a Björk-Shiley mitral valve prosthesis. Thorax $1983 ; 38: 390-1$.
a

a Björk-Shiley mitral valve prosthesis. Thorax 1983;38:390-1.
7 Ibarra F, Gutierrez A, Martinez F, Carreras L, Lopez C, Alonso-Lej F. Ibarra F, Gutierrez A, Martinez F, Carreras L, Lopez C, Alonso-Lej F.
Fracture of the outlet strut of a Björk-Shiley mitral prosthesis: emergency operation with survival. $J$ Thorac Cardiovasc Surg 1984;87:315-8.

8 Jaumin P, Ponlot R, Schoevaerdts J-C, Brasseur L, Chalant C-H. Strut fracture of the Björk-Shiley aortic valve. J Thorac Cardiovasc Surg 1984;88:787-91.

9 Advising patients with artificial heart valves. Lancet 1990;336:152.

10 Taylor K. Acute failure of heart valves. $B M J 1988 ; 297: 996-7$.

11 Sethia B, Turner MA, Lewis S, Rodger RA, Bain WH. Fourteen years' experience with the Björk-Shiley tilting disc prosthesis. J Thorac Cardiovasc Surg 1986;91:350-61.

12 Treasure T. Which heart valves should we use? Lancet 1990;336:1115-7.

13 Collins JJ. The evolution of artificial heart valves. $N$ Engl J Med 1991;324:624-6. 